\title{
Editorial of Concern
}

\author{
Sara H. Sadok ${ }^{1}$ Rayssa L. Borges-Medeiros ${ }^{1}$ · João R. M. de Oliveira ${ }^{1}$
}

Received: 15 August 2021 / Accepted: 20 August 2021 / Published online: 3 September 2021

(c) The Author(s), under exclusive licence to Springer Science+Business Media, LLC, part of Springer Nature 2021

\section{Dear Editors,}

We would like to share an urgent concern regarding SARSCoV-2 and hyper-immunoglobulin E syndrome (hyper-IgE syndrome, Job disease), besides common polygenic carriers of thalassemia, sickle cell anemia traits, and other underdiagnosed conditions of genetic origin that might be misleading the current studies of risk groups and the actual hallmarks of SARS-CoV-2.

In our study of cases in an adult Brazilian population that included the collection of clinical findings such as deep phenotyping, blood tests, mental status, metabolic profiles, and immune-related disorders including IgE dosage, it has come to our attention that Job Disease may present as a spectrum of mild symptomologies. Job disease (hyper-IgE syndrome related to STAT-3 variants) is characterized by chronic lung infections, otitis, high palate, asthma, eczema, encephalopathies, bone deformities, and predisposition for various types of malignancies. In the classical clinical presentations, the symptoms are severe and the condition is often diagnosed during neonatal period or early childhood. We noted in our analyses of adults that many of the mild multisystemtic features of hyper-IgE are shared by individuals of, for example, Mediterranean background, African ancestry, and high inbred populations. Besides, we also found various cases with carriers of beta thalassemia and sickle cell anemia traits that may have been overlooked until now as risk factors for comorbidities. We hypothesize that these conditions, especially hyper IgE, unlike the severe presentations seen in newborns and children, are underdiagnosed in adult groups due to mild symptoms, and suggest that hyper-IgE spectrum may not be rare among Brazilian adults and hence very likely not rare in Portugal, France, Spain, Italy, Israel, and other affected populations.

We are concerned that (1) undiagnosed lung lesions in the patients with mild hyper-IgE spectrum may be exacerbated by SARS-CoV-2, and (2) patients with mild hyper-IgE spectrum, thalassemia, and sickle cell anemia may be a large unrecognized high SARS-CoV2 risk groups.

In an ongoing pandemic, patients with hyper-IgE syndrome and other overlooked conditions may be more susceptible to the grim outcomes of the viral infection; hence, it would be extremely important to confirm this hypothesis as it may modify and improve screening procedures and treatments.

Acknowledgements We would like to thank the following funding agencies: CNPq, Capes, Facepe, Propesq- UFPE, Decit-MS, John Simon Guggenheim Memorial Foundation, and patients involved in the studies during all the years of duration.

We would like to express our gratitude to Denis Moura, Gustavo de Lima Cavalcanti, José Eriton Gomes, Laura Botler and Silvana Santos.

Publisher's Note Springer Nature remains neutral with regard to jurisdictional claims in published maps and institutional affiliations.

João R. M. de Oliveira

joao.ricardo@ufpe.br

1 Laboratório de Imunopatologia Keizo Asami, Federal

University of Pernambuco, Recife, Pernambuco 50670-901,

Brazil 